\title{
Confianza política y medios de comunicación. Teoría, hallaz- gos y metodologías
}

Political trust and media. Theory, findings and methods

\author{
Evelia Mani \\ Benemérita Universidad Autónoma de Puebla \\ Orcid https://orcid.org/oooo-0oo2-8098-4323 \\ evelia.mani@gmail.com
}

\author{
Martín Echeverría \\ Benemérita Universidad Autónoma de Puebla \\ Orcid http://orcid.org/oooo-0oo1-6071-8725 \\ echevemartin@yahoo.com.mx
}

Resumen: En el contexto de la relevancia de la confianza política para la gobernabilidad democrática, se ofrece un estado del arte basado en 52 trabajos publicados en los últimos diez años en bases de datos latinoamericanas e internacionales, acerca del factor mediático implicado en ello. El documento reseña la función de la confianza para la democracia y las principales teorías que explican su variación temporal. Centrándose en la explicación específicamente mediática, se explicitan las vertientes clásicas y contemporáneas de los efectos de los medios a este respecto, así como los trabajos empíricos que las apoyan o refutan. El artículo concluye con una reseña de las principales metodologías implementadas para su estudio, así como una reflexión crítica acerca de las lagunas de conocimiento e insuficiencias encontradas en la revisión, como punto de partida para nuevas pesquisas.

Palabras clave: Confianza política, medios de comunicación, videomalestar, efectos de los medios, metodologías.

\begin{abstract}
In the context of the relevance of political trust to democratic governance, we present a literature review based on 52 papers published in the last ten years in Latin-American and international databases about the media factors implied in that subject. The paper describes the function of trust to democracy and the main theories that explain its temporary variations. Focusing on the media explanation only, we describe the classic and contemporary perspectives of media effects about it, as well as the empirical work that supports or contend it. The paper concludes with a synthesis of the main methods applied in the literature, and a critical appraisal about knowledge lacunae and deficiencies found in the review, as antecedents of new research.
\end{abstract}

Keywords: Political trust, media, videomalaise, media effects, methods.

Fecha de recepción: 31/07/2019

Fecha de aprobación: 19/09/2019

Cómo citar este artículo / How to cite this paper: Mani, E., \& Echeverría, M. (2019). Confianza política y medios de comunicación. Teoría, hallazgos y metodologías. Revista de Comunicación Política, 1, 53-74. doi:10.29105/rcp1-3 


\section{Introducción}

Confiar en quienes gobiernan es un componente importante para la funcionalidad de la democracia, en tanto permite márgenes de acción programática, legitimidad y gobernabilidad de parte de quienes ejercen el poder. Aunque una dosis de sana desconfianza e incluso escepticismo también es recomendable en vistas a una ciudadanía crítica y menos manipulable, el apoyo a los actores e instituciones que gobiernan, así como la vigencia del contrato social entre el pueblo soberano y los gobernantes, son un activo valioso para resolver problemas de política pública y en última instancia consolidar un régimen (Hetherington, 1998).

Se trata no obstante de un activo a la baja. Diversos estudios constatan el sostenido decremento de la confianza en el campo político en todo el mundo: los políticos como individuos, los partidos políticos, el gobierno, el sistema político, e incluso el régimen democrático, han pasado de una confianza manifiesta del 71\%, en 1956, al 22\% en el año 2012 tan sólo en Estados Unidos y del 52\% al 37\% en Canadá durante el mismo periodo (Dalton, 2017, pp. 377-378). Desde una óptica histórica el enorme consenso de las élites y de los ciudadanos surgido de la posguerra se ha ido disipando, al tiempo que el sentimiento popular de animadversión generalizada -sin distingo de filiaciones partidistas o niveles de gobierno- se ha intensificado: de una desconfianza funcional a un escepticismo acendrado y de ahí al cinismo o predisposición negativa a priori hacia el campo político, con imputaciones de falta de atención, ineptitud y corrupción por parte de los políticos (Herman, 2010).

El fenómeno, ciertamente inquietante, recibe algunas explicaciones en la literatura: postula por un lado una mayor complejidad de los sistemas políticos en los que el gobierno de una tecnocracia le es esotérica y suspicaz al grueso de la población; la constante incertidumbre económica que deja al ciudadano en un ambiente económicamente inestable y con la certeza acerca de la impotencia de los poderes públicos para controlar la economía; la dilución de la cohesión social en favor del anonimato individualista (Rosanvallon, 2006); o la pérdida de un referente ideológico que otrora construía lealtades fuertes entre los ciudadanos hacia los partidos políticos, lo que ha dificultado una adhesión significativa de los mismos (Cueto, 2007). No obstante, un factor que a menudo se considera también de relevancia es el de los medios de comunicación: el supuesto principal es que la mediación pública de la realidad política, sobre todo en un tono negativo, tiene consecuencias cognitivas en las actitudes de la opinión pública hacia los actores e instituciones democráticas, en un momento histórico en el que la principal fuente de acceso al conocimiento político son los medios de comunicación. Estas consecuencias pueden ser negativas, en el sentido de minar la confianza ciudadana en los políticos y generar cinismo hacia los mismos, o bien positivas, en el sentido de ser capaces de movilizar políticamente a los ciudadanos.

La siguiente revisión de literatura se propone sintetizar y actualizar este debate, avanzando en algunos aspectos que consideramos deficientes en cierta parte de la literatura científica al respecto. Para tal finalidad, exploramos la literatura académica publicada entre los años 2007 y 2017 por medio 
de la base de datos Scopus bajo las palabras clave "confianza política” y “efectos de los medios", de lo cual se obtuvieron 52 documentos revisados para este artículo. Por la naturaleza de estado del arte de este ejercicio, la búsqueda incluyó fundamentalmente estudios empíricos, dejando de lado ensayos que seguramente hubieran incrementado el número de documentos. Cabe señalar que la mitad de las entradas provienen de Estados Unidos y en Scopus no se reporta ningún documento de Latinoamérica, por lo que la búsqueda para esta área geográfica se realizó en Redalyc, donde Ecuador, México y Colombia lideran las investigaciones sobre el tema. Para fines de este documento, se revisaron aquellos textos en las disciplinas de ciencia política y de comunicación política que trataran la relación entre los medios de comunicación y confianza política.

Respecto al orden de exposición, en primera instancia exploramos la relevancia de la confianza política y la manera en que ésta contribuye a la funcionalidad de un régimen democrático, así como sus principales explicaciones. Posteriormente vinculamos la mediación cognitiva de los medios de comunicación con el cultivo de actitudes en general y de la confianza política en particular; revisamos las teorías o hipótesis centrales acerca de los efectos mediáticos en la desconfianza, así como los apoyos empíricos que han ganado recientemente; y reseñamos someramente los diseños y prácticas metodológicas que se han utilizado para abordarlos. Finalizamos con un balance de las posiciones encontradas, así como un señalamiento de inconsistencias y lagunas de conocimiento a atender. Se ofrece, pues, un compendio y puesta al día del conocimiento científico al respecto, que pretende convertirse en un insumo de partida para investigaciones dedicadas hacia dicho tema.

\section{Confianza política. Definición y relevancia en el régimen de- mocrático}

Como actitud humana innata, la confianza trasciende el ámbito social cuando las interacciones cotidianas ya no pueden resolverse de forma interpersonal y requieren trasladarse a un ámbito normativo como el de la política. Debido a que la política se distingue de otras relaciones sociales por llevar implícito un conflicto de interés cada que se enfrenta a la resolución de cualquier asunto relacionado con la acción colectiva, el significado de la confianza y su papel dentro de este entorno reviste un significado particular (Warren, 1999a). Diversos autores coinciden en definir a la confianza política como el reflejo de la evaluación ciudadana respecto al funcionamiento de los actores políticos y las instituciones (Blind, 2007; Hakhverdian \& Mayne, 2012; Hetherington \& Husser, 2012; Knorn, 2016; Levi \& Stoker, 2000). Dicha evaluación se realiza comparando qué tanto se corresponde el desempeño de actores e instituciones políticas con las expectativas de la sociedad, particularmente respecto a su competencia, fiabilidad, así como su preocupación por atender los intereses de quien confía en él (Levi \& Stoker, 2000). En esta relación, los ciudadanos son vistos como los sujetos que confían y las instituciones y sus actores como los objetos sobre los que se deposita la confianza, que en última 
instancia los libera de la preocupación y de la necesidad de monitorear parcial o totalmente el comportamiento de aquel en quien confían.

La confianza política tiene una relevancia fundamental en las democracias y sus instituciones, al constituir "un indicador central del sentimiento subyacente del público sobre su sistema político" (Newton \& Norris, 2000, p. 53), que cristaliza el estado del contrato social asumido entre los ciudadanos y su gobierno (Dalton, 2017). Así, la confianza presenta implicaciones lo mismo en instituciones políticas, ciudadanos, que el régimen mismo.

En primer lugar, las instituciones gubernamentales se benefician de la confianza de sus ciudadanos para ser más eficientes y desde luego legítimos (Blind, 2007; Gómez, 2011; Warren, 1999b). La evidencia empírica demuestra que la confianza se traduce en aprobación hacia los gobiernos, lo cual a su vez les otorga un mayor margen de libertad y maniobra para gobernar -como en la resolución pacífica de conflictos- así como un mayor apoyo a sus decisiones, con lo cual pueden funcionar con fluidez y efectividad (Bauer \& Fatke, 2014; Hetherington, 1998).

Por parte de los ciudadanos, la confianza en los funcionarios y sistemas legales los impulsa a cumplir sus deberes cívicos de manera voluntaria, incluidas las leyes, regulaciones gubernamentales y obligaciones ciudadanas, como pagar sus impuestos (Hakhverdian \& Mayne, 2012; Seyd, 2016). También les ayuda a reforzar la creencia de que el gobierno tiene capacidad de respuesta, por lo tanto, pueden estar seguros de que sus demandas son escuchadas y atendidas, lo cual podría eventualmente incentivar la participación política (Levi \& Stoker, 2000; Mishler \& Rose, 2005). En contraparte, la falta de un vínculo entre ciudadanos e instituciones generada por la desconfianza puede tener como consecuencia la autoexclusión o incomprensión de los asuntos políticos, baja o nula participación en los procesos democráticos y apatía, cinismo o desinterés, aspectos que, a la larga, pueden incidir de manera negativa tanto en el tejido social como en el desarrollo de la democracia (del Tronco, 2012; Morales, 2008).

De igual manera, la confianza en las instituciones democráticas redunda en el apoyo ciudadano a los principios y al régimen democrático, su legitimidad y estabilidad, e implícitamente un rechazo hacia alternativas no democráticas (Hakhverdian \& Mayne, 2012; Mishler \& Rose, 2005). Particularmente en regímenes de democracia representativa, los ciudadanos que delegan poder sobre instituciones y representantes políticos actúan de buena fe sobre la base de confiar en quienes los representan. En consecuencia, la baja confianza en esos representantes pudiera impactar en la baja confianza y legitimidad del régimen en su conjunto.

En contraparte, otra corriente estima a la desconfianza como un elemento saludable para las democracias, en cuanto protege al individuo del poder público y busca evitar el despotismo y el abuso de poder presente en otras épocas (Rosanvallon, 2007). Una cierta dosis de desconfianza implica además que los ciudadanos han superado su ignorancia, ingenuidad y deferencia, manifestando en cambio un escepticismo crítico indicativo de una sociedad informada que ha alcanzado cierta madurez 
política, capaz de cuestionar, participar y tomar decisiones (Dogan, 2005, p. 45). La vigilancia y evaluación ciudadanas, el control y la distribución de poder, así como las libertades de protesta y organización social, son manifestaciones institucionales de una desconfianza que deviene funcional para los sistemas democráticos (Warren, 1999b).

Finalmente, y a pesar de su relevancia, el estado de la confianza pública a nivel internacional es más bien precario: independientemente del sistema electoral, de su historia o las circunstancias actuales de cada país, la confianza hacia la política y sus instituciones muestran una disminución importante desde hace algunos años, afectando a la mayoría de las instituciones y actores políticos o gubernamentales (Catterberg \& Moreno, 2006; Hetherington \& Rudolph, 2008; Knorn, 2016; Levi \& Stoker, 2000). Esto sucede tanto en los Estados Unidos, cuya caída comenzó en el último tercio del siglo XX de la mano de la crisis económica y los escándalos políticos (Kanji, 2002), como en Europa, a nivel de prácticamente todas las instituciones públicas (Dogan, 2005). Las democracias latinoamericanas no escapan a estas tendencias, pues las mediciones de Latinobarómetro, desde 1995, han demostrado durante dos décadas un descenso continuado de la confianza en las instituciones políticas, siendo de las más bajas del mundo (Latinobarómetro, 2016).

No todos los entes políticos son afectados por igual en las cuotas de desconfianza. Los objetos del nivel macro -la confianza difusa en el sistema político democrático- no registran niveles de desconfianza tales que puedan generar crisis sistémicas agudas; a lo sumo existen fluctuaciones en dichos niveles de confianza cuya intensidad y duración en el tiempo varía (Norris, 2011), aunque dicha insatisfacción no llega a afectar negativamente la adhesión a los principios democráticos (Dalton, 2017; Zmerli \& van der Meer, 2017). En las antípodas, los partidos políticos son los que "generan mayor desconfianza de entre todas las instituciones y organizaciones políticas de todos los continentes y tipos de regímenes" (Dogan, 2005, p. 23); a su vez, líderes o figuras políticas también salen desfavorecidas, puesto que "en las encuestas internacionales de ética profesional relativas a la honestidad de unas veinticuatro profesiones, los políticos aparecen en muchos países como los menos dignos de confianza" (Dogan, 2005, p. 26). En ello interviene el desgaste natural que tienen los líderes en el ejercicio de gobierno, una legitimidad restada por el hecho de haber sido elegidos por mayorías no siempre amplias, así como la dificultad de comunicar su quehacer a todos los ciudadanos representados, particularmente cuando su mensaje está mediado por sus propios partidos, el ente de gobierno al que pertenecen y los medios de comunicación. A este punto regresaremos en el siguiente apartado.

\section{Factores que impactan en la confianza política y el papel de los medios de comunicación}

La pregunta central es, pues, qué factores han originado este declive en la confianza política. La determinación de los mismos pasa necesariamente por una revisión de dos tradiciones teóricas: la teoría 
institucionalista (Coleman, 1986; Hetherington \& Rudolph, 2008; Jackman \& Miller, 1996) y la culturalista (Almond \& Verba, 1963; González, 2006; Morales, 2008; Putnam, Leonardi, \& Nanetti, 1994). La primera indica que la confianza es endógena y está asociada al desempeño de las instituciones: por lo tanto, ésta dependerá entonces de la utilidad reportada a los individuos a partir de la actuación satisfactoria de aquellas. Por otro lado, para la teoría culturalista la confianza es exógena, lo que significa que se origina fuera de la esfera política, a partir de las normas transferidas hacia los individuos en el proceso de socialización. Se trata de una extensión de la confianza personal que, una vez influenciada por las normas arraigadas en la cultura, se proyecta en las instituciones.

La investigación del desempeño institucional como variable explicativa endógena está centrada sobre todo en la importancia de los aspectos económicos: los ingresos, niveles de desempleo e inflación resultan determinantes para que los ciudadanos emitan un juicio sobre el trabajo de sus políticos y gobierno, para cuidar de los intereses y el bienestar de la sociedad a la que representan (Hetherington \& Rudolph, 2008), por lo que se espera que niveles altos de confianza estén positivamente relacionados con aquellos ámbitos donde el gobierno puede generar crecimiento económico (Blind, 2007). Las evaluaciones del desempeño político también son relevantes para que los ciudadanos confíen, sobre todo en aspectos como la protección de las libertades civiles, el respeto al Estado de derecho y sobre todo la integridad política respecto a la corrupción gubernamental (Mishler \& Rose, 2005), a la que los ciudadanos están atentos en prácticamente cualquier país (Blind, 2007)

Por su parte, entre los factores exógenos que explican sobre todo la desconfianza se encuentra la globalización. La necesidad por parte de las naciones de formar parte de una sociedad global ha restringido el poder de los gobiernos sobre sus condiciones sociales y económicas. Muchas políticas son moldeadas por acuerdos internacionales y estas restricciones impuestas a los gobiernos contribuyen a la erosión de la confianza de sus ciudadanos hacia ellos (Dalton, 2017). El manejo de los gobiernos respecto de los factores macroeconómicos es una muestra de ello, pues éstos ya no ofrecen proyecciones fiables a medio y largo plazo, dejando al ciudadano en un ambiente económicamente inestable y proyectando la impotencia de los poderes públicos para controlar la economía. Este aspecto también se vincula al ascenso de la tecnocracia en las democracias modernas, donde los expertos ejercen poder con sus conocimientos inaccesibles para el ciudadano promedio (Rosanvallon, 2006).

Otro factor exógeno que influye en la confianza política es la fluctuación del capital social (Dalton, 2017). En aquellas sociedades donde son más fuertes los lazos entre individuos y comunidades, se suele confiar más en el gobierno. Sin embargo, la movilidad social y geográfica presente en muchos países evita relaciones sociales estrechas que puedan concretarse en participación social y compromiso cívico, por lo que, si las personas no confían en su círculo más cercano, dicha desconfianza se extenderá a sus representantes en el gobierno (Blind, 2007). 
Finalmente, otras líneas de investigación de tipo exógeno consideran fundamental el papel de los medios de comunicación, generalmente como sospechosos o responsables en el declive de la confianza hacia las instituciones y actores (Loveless, 2010), así como del cinismo y la separación de los ciudadanos respecto de la política (Botan, Corbu, \& Sandu, 2016). La importancia de las instituciones mediáticas en el fenómeno se basa en supuestos de tipo cognitivo y actitudinal respecto a la política. Por un lado, es un hecho que la mayoría de la gente depende de los medios para informarse sobre política, sobre las propuestas y acciones de los políticos, así como los hechos y las opiniones críticas hacia los mismos; funcionan, en efecto, como conductos de información política (Perloff, 2013). Pero, por otro lado, los medios dan forma a lo que los ciudadanos opinan y sienten sobre un tema, estimulan a los mismos "a evaluar la calidad de la gobernabilidad democrática (...) enmarcando si el desempeño del gobierno se percibe positiva o negativamente en contra de estos" (Norris, 2011, p. 9).

Este panorama, en el que la política está cada vez más mediatizada (Strömbäck \& Shehata, 2010), genera una preocupación creciente respecto al papel de los medios de comunicación y cómo su contenido y su uso se relacionan con la afectación de los niveles de confianza en las instituciones políticas y la democracia, que resulta en ocasiones negativa, otras positiva y otras moderada por variables externas, como se reseña en la siguiente sección.

\section{Teorías y estudios empíricos contribuyentes}

Los efectos de los medios de comunicación aplicables a la confianza política se distribuyen en dos grandes corrientes teóricas. Por un lado, encontramos a aquellos autores que atribuyen a los medios de comunicación y sobre todo a la presentación negativa de la política, el provocar desafección, cinismo y apatía entre los ciudadanos (Luengo \& Maurer, 2009). Sin embargo, otro importante número de estudios ha encontrado evidencias de efectos que ayudan a los ciudadanos a mantenerse informados y comprometidos, estimulando su participación y movilización en las actividades políticas.

\section{Videomalestar}

El término videomalestar fue acuñado por Robinson en los años setentas para describir el "fenómeno dual de la pérdida de confianza en las instituciones políticas y el incremento de la dependencia de los individuos a la televisión como un medio para obtener información política" (Holtz-Bacha, 1990, p. 73). En consonancia con esta idea, Moy y Scheufele (2000) indican que, por ejemplo, el incremento de la penetración de la televisión en los hogares de Norteamérica ha sido asociado con la disminución de la participación electoral y con bajos niveles de conocimiento político. 
Robinson, por su parte, argumentó que las transmisiones televisivas bombardeaban a sus audiencias con "noticias interpretativas, sensacionalistas, agresivas y anti institucionales" (Robinson, 1976, p. 426). Esta práctica fue considerada como la responsable del incremento de un malestar hacia la política, ya que al carecer de sofisticación y verse expuestas a noticias con alto grado de negatividad, enmarcadas en el conflicto y envueltas de críticas desfavorables por parte de los periodistas, las audiencias se hacían más cínicas, frustradas, desesperadas y cada vez menos comprometidas con las instituciones.

A esta línea sobre los efectos perniciosos de los medios de comunicación y específicamente de la televisión, se adhirieron diversos trabajos (Cappella \& Jamieson, 1996; Hibbing \& Theiss-Morse, 1995; Iyengar \& Kinder, 2010; Patterson, 1993), elaborados sobre la base de la teoría del framing o encuadre, en la cual se asume que la forma de presentar los acontecimientos y temas políticos puede influir, más que en la percepciones, en la manera en que las audiencias los comprenden, e incluso en sus actitudes políticas (Aruguete \& Muñiz, 2012).

$\mathrm{Al}$ enmarcar la política en términos estratégicos, centrarse en las tácticas o describir como egoístas los motivos de los políticos, los medios han sido responsabilizados de cultivar actitudes cínicas que alimentan el descontento público y la desvinculación con la política (De Vreese \& Elenbaas, 2008). Las investigaciones recientes apuntan en su conjunto a la posición de que el impacto de los encuadres utilizados para presentar información política en la disminución de la confianza es provocado por el énfasis que hacen algunos medios en los desacuerdos entre los partidos políticos y, de manera más amplia, en la ineficiencia percibida de las instituciones políticas. Esto tiene un poderoso efecto negativo en las instituciones políticas que en lugar de ser concebidas como eficientes, se ven socavadas no sólo en la percepción de su quehacer, sino también en la imagen de sus líderes (Newton, 2006).

En esa línea, Valentino, Buhr, \& Beckmann (2001) encontraron que el uso de encuadres estratégicos provocaban reacciones negativas hacia los políticos y hacían que la retención de información fuese menor; Chen \& Shi (2001) demostraron que en China los medios de comunicación tienen efectos negativos sobre las actitudes de la gente hacia las instituciones políticas en general y provocan desconfianza en el gobierno; los hallazgos de Mutz \& Reeves (2005) sugieren que aunque la incivilidad en el discurso público provoca el interés de los espectadores, afecta negativamente la confianza que éstos tienen en el gobierno. Kleinnijenhuis, Van Hoof y Oegema (2006), por su parte, también encontraron que las noticias negativas tenían un efecto significativo en la confianza en los líderes de los partidos políticos; Boţan (2013) reporta que los conflictos políticos encuadrados de forma incivil contribuyen significativamente a la disminución de la confianza política.

Más allá de las noticias emitidas, otra línea de investigación vincula efectos de desconfianza con el consumo de contenidos de entretenimiento: advierte que la cobertura centrada en el entretenimiento puede debilitar la confianza política y que el creciente uso de fuentes de noticias no tradicionales (Guggenheim, Kwak, \& Campbell, 2011) o bien la exposición a programas de comedia política 
(Tsfati, Tukachinsky, \& Peri, 2009) pueden disminuir los niveles de confianza o fomentar actitudes de desconfianza.

Otros estudios buscan explicaciones sobre la desconfianza en función de condiciones antecedentes de emisión mediática, tales como las fuerzas económicas y políticas que rigen la producción noticiosa. Tal es el caso del estudio de Ariely (2015), que analiza el papel de la creciente comercialización de los medios sobre la influencia negativa hacia la cultura política. Su análisis, que abarca a 33 países europeos, destaca que "independientemente de las diferencias entre los modelos de medios de comunicación europeos, la comercialización de su entorno (...) está relacionada con niveles más bajos de confianza política” (p. 439), confirmando que los efectos negativos de la comercialización en los medios trascienden a Estados Unidos y también ocurren en Europa (Ariely, 2015).

Respecto a la literatura latinoamericana, Pereira (2000) introduce al debate la incidencia de los medios de comunicación en la crisis de confianza en la política y sus instituciones para el caso brasileño. El autor analiza los fenómenos referentes a la ofensiva del periodismo, la "antipolítica" de los medios de comunicación y el carácter sesgado de la cobertura periodística de las instituciones políticas. Concluye que existe evidencia suficiente de que la cobertura mediática genera serios obstáculos a la legitimidad democrática, especialmente para el poder legislativo.

En el caso mexicano, Moreno (2008) revisa el impacto de las noticias sobre la confianza política, mediante un diseño tipo panel de dos rondas de entrevistas, una inmediatamente antes de la campaña presidencial y la otra inmediatamente después de los comicios. Sus resultados destacan la polarización política vivida durante esas elecciones en México y cómo permeó la desconfianza en las instituciones electorales.

Si bien los estudios sobre malestar mediático obtuvieron resultados significativos que comprobaban sus supuestos, también surgieron y prevalecen investigaciones en dirección opuesta que arrojan dudas sobre la aplicación de esa perspectiva teórica a todos los casos de exposición mediática y sus efectos.

\section{Teorías de la movilización}

Las teorías de la movilización política nacieron como contraste a las hipótesis del malestar mediático y sugerían que los consumidores de noticias televisivas y lectores habituales de prensa política estarían más inclinados a querer informarse, interesarse y comprometerse con la vida política, independientemente del tono de la cobertura mediática (García \& Ruiz, 2007). A contrapelo de la teoría del malestar mediático, esta nueva interpretación sugiere que el impacto de los medios tiene más bien una dirección positiva al promover la participación democrática. Aunque el concepto no incluye entre sus dimensiones el fenómeno de la confianza, pues la participación es más bien un patrón de conducta, dicho constructo está implicado en su modelo de manera indirecta; con todo, lo relevante es la 
manera en que desautoriza la hipótesis del videomalestar como la causación dominante dentro de estas problemáticas.

Así, Holtz-Bacha (1990) realizó uno de los primeros trabajos en los que se plantea que existe un efecto positivo de los medios de comunicación. En su investigación concluye que "la relación de la exposición a la información política en la televisión y en los medios impresos se vincula tanto con una visión menos alienada de la política como con niveles más altos de participación” (Holtz-Bacha, 1990, p. 83), descartando así la teoría del videomalestar, por lo menos para el caso de Alemania. En este mismo sentido, Newton (1999) encontró que en Gran Bretaña los lectores de periódicos de gran formato mostraban un mayor conocimiento, interés y confianza política y eran menos cínicos, mientras que ver mucha televisión no estaba asociado con la desconfianza política o falta de respeto por la democracia británica.

Siguiendo esta línea de los efectos positivos, Norris (2000) analiza datos provenientes de 28 democracias occidentales y, dados sus hallazgos, propone la existencia de un círculo virtuoso entre consumo mediático y el compromiso político, asegurando que "el uso de los medios de comunicación se asocia positivamente con una amplia gama de indicadores de conocimiento político, confianza y movilización” (Norris, 2000, p. 17).

El planteamiento central de la teoría del círculo virtuoso es que los ciudadanos más expuestos a los medios de comunicación desarrollan mayor compromiso y confianza política. Una vez que se confía en la política, el interés en las noticias se incrementa, surgiendo así una relación recíproca entre el uso de medios y la confianza política que se incrementa a través de tiempo. En palabras de Norris (2000), "los más políticamente informados, confiados y participativos son más propensos a sintonizar la cobertura de asuntos públicos. Y los más atentos a la cobertura de los asuntos públicos se involucran más en la vida cívica” (p. 17). En el otro extremo, los no comprometidos difícilmente se expondrán a contenidos políticos ni les prestarán atención, debido a que desconfían de los contenidos ofrecidos por los medios, por lo que éstos no tendrán ningún impacto en este segundo grupo.

Esta teoría también ha presentado importante evidencia en el campo empírico, que sugiere que los usuarios regulares de noticias de televisión normalmente muestran actitudes más positivas hacia la política y el gobierno, y que el interés político previo y el conocimiento estimulan la exposición y la atención a las noticias. Este proceso, a su vez, refuerza gradualmente el conocimiento práctico, la confianza política y el activismo cívico.

En su análisis, que abarca distintas fuentes noticiosas y utiliza sondeos de opinión, Moy y Pfau (2000) encontraron que los medios impresos eran más eficaces que las noticias televisivas o radiofónicas para aumentar los niveles de confianza. Otra investigación que apoya esta idea es la de Aarts y Semetko (2003) quienes confirmaron que la exposición a los programas noticiosos de televisión pública parecía tener un impacto positivo en la evaluación de los principales partidos políticos, y que el uso de los medios puede estar claramente relacionado con la participación política. 
Ulbig y Dunaway (2008) realizan una distinción interesante sobre cómo los medios afectan de forma diferente las actitudes hacia los actores políticos nacionales y subnacionales, demostrando que el consumo de noticias parece no tener un efecto apreciable sobre la confianza en el gobierno nacional, pero afecta la confianza en el gobierno estatal. En este mismo sentido, Yang, Tang, Zhou y Huhe (2014) encuentran que el consumo de medios incrementa la confianza en instituciones de alto perfil como el gobierno central y el Partido Comunista, y disminuye la confianza en instituciones de bajo perfil, tales como los gobiernos locales, la administración pública, los tribunales y la policía.

En un trabajo posterior que incluía a Reino unido, Estados Unidos y cuatro países más de Europa continental, al medir el impacto de la exposición a las noticias sobre la confianza, Aarts, Fladmoe y Strömbäck (2010) encontraron principalmente efectos positivos en los países corporativos democráticos, es decir aquellos ubicados en Europa del Norte y Central, así como algunos países del mediterráneo, cuyo sistema de medios comparte un alto paralelismo político, un alto nivel de profesionalización periodística y límites al poder estatal que permiten una mayor libertad de prensa (Hallin \& Mancini, 2004).

Por su parte, Shen y Guo (2013), así como Luengo y Coimbra-Mesquita (2013), encontraron que el consumo de noticias televisivas se asoció positivamente con la confianza política en China y Brasil, respectivamente. Por su parte, Camaj (2014) explica que los medios de comunicación resultan beneficiosos para el proceso de democratización, ya que contribuyen a la formación de actitudes de los ciudadanos que no son necesariamente negativas respecto a las instituciones políticas. Por su parte, Strömbäck, Djerf-Pierre y Shehata (2016) realizan una investigación longitudinal donde analizan la relación entre el uso de los medios de comunicación y la confianza política, cuyos resultados demuestran un vínculo positivo entre ambos.

En el ámbito latinoamericano, una buena parte de los trabajos apoyan también una visión virtuosa de la exposición a medios. García y Ruiz (2007) comparan la conexión entre el activismo político, como una de las dimensiones de la desconfianza política, y los medios en España y México, encontrando evidencia de que los medios provocan movilización. Sus hallazgos también señalan las marcadas diferencias en los grupos que se exponen en menor medida a los medios, y los que dicen no acercarse nunca a los medios para obtener información política.

Por su parte, Moreno (2010) coordina un libro en cuyos capítulos se analiza la confianza política depositada en diversos actores e instituciones. Al hacer una recapitulación de sus hallazgos, este autor explica que de entre las variables que resultaron estadísticamente significativas, la primera que aparece es el grado de exposición a medios de comunicación, y explica la confianza en 15 de las 17 instituciones estudiadas, arrojando coeficientes con signo positivo, confirmando "que a mayor exposición a medios mayor confianza en las instituciones" (2010, p. 46).

También en el caso mexicano, Corduneanu \& Muñiz (2011) exploran la influencia que la exposición a los medios de comunicación genera sobre las actitudes de la población como la confianza 
política, el interés en la política, la eficacia política y la valoración de la democracia, cuyos resultados apoyan la teoría de la movilización, ya que, de acuerdo con ellos, la televisión generó en mayor nivel de confianza hacia los actores políticos. Asimismo, Guerrero, Rodríguez-Oreggia y Machuca (2014) encuentran que el consumo de información por televisión tiene un efecto significativo y positivo en casi en todas las variables de cultura cívica, incluida la confianza política, pero también una relación negativa entre el uso de Internet y la confianza que en el caso específico de las elecciones de 2006.

Finalmente, Díaz (2017) introduce a la confianza política como uno de los componentes del compromiso político, al cual analiza en el contexto de la elección presidencial de 2012. Sus resultados apoyan la teoría de la movilización al encontrar que la exposición a la información de la campaña en medios de comunicación no tuvo un impacto negativo.

$\mathrm{Al}$ encontrar evidencia empírica tanto para la teoría del videomalestar, como para la de movilización, y confirmar que los resultados no pueden considerarse concluyentes sólo para una de ellas, se plantea la necesidad de enriquecer las explicaciones sobre la influencia de los medios bajo la premisa de que la exposición a éstos desalienta la confianza política bajo ciertas condiciones, pero también la promueve bajo otras (Newton, 1999). Por esta razón, otro cúmulo de trabajos exploran aquellos otros factores intervinientes en esta relación.

\section{Efectos moderadores y factores intervinientes}

En muchos casos, se encontró que los resultados que apoyaban la influencia positiva o negativa de los medios sobre la confianza política estaban moderados por factores personales como, entre otros, el interés político, las redes sociales y los valores personales otros (Newton, 2006), así como por las características de las fuentes de noticias (Avery, 2009), lo que constituyó un refinamiento para el debate teórico y los estudios empíricos que ya no se reducirían a concluir si los medios incidían positiva o negativamente, sino que analizarían cómo la influencia de distintas variables podía moderar los efectos mediáticos.

Respecto a los factores personales que actúan como moderadores, los resultados de la investigación de Wilkins (2000) indican que la televisión por sí sola no explica que surja desconfianza política, pero la lectura de periódicos y el consumo de noticias televisivas por parte de quienes tienen un nivel de educación alto, tienden a asociarse con mayor participación. Floss (2008) argumenta que el impacto de los medios masivos en las actitudes políticas, como la confianza, es moderado por las preferencias políticas de los ciudadanos. Ceron y Memoli (2015) aseguran que los medios de comunicación sólo refuerzan las actitudes preexistentes y que incluso si la información en ellos es contraria a las actitudes de quienes los consumen, esto apenas altera la confianza en el gobierno, lo que coincide con la idea de que cuando las personas tienen una tendencia inicial hacia el cinismo, los medios tendrán efectos negativos en ellas (Degirmenci, 2016). 
En esta línea de investigación también cobra importancia la necesidad de explicar que los efectos de los medios se matizan de acuerdo con los formatos y características de éstos (Lee, 2005), tomando en consideración que las fuentes de información se han diversificado considerablemente en las últimas dos décadas. Avery (2009) sostiene que la influencia de los medios de comunicación en la confianza política varía si la fuente de noticias es la prensa o la televisión. Schmitt-Beck (2015) confirman para el caso alemán que la exposición a periódicos de gran formato estimula la participación, no así tratándose de la televisión; Strömbäck et al. (2016) encuentran que la relación entre el uso de medios informativos y la confianza es positiva cuando se trata de periódicos matutinos y noticias de televisión de servicio público, y no es significativa tratándose de noticias de televisión comerciales.

Para el caso mexicano, Aruguete y Muñiz (2012) encuentran una relación significativa entre la conversación política, el consumo de ciertos medios de comunicación y las actitudes políticas por parte de la población. Aseguran que en el proceso democratizador mexicano los medios de comunicación han jugado un papel crucial, como sucedió en la elección de 2006. Entre sus hallazgos destaca la relación significativa entre las variables conversación política, consumo de ciertos medios de comunicación y las actitudes políticas.

Con estos esfuerzos el debate sobre la relación entre los medios de comunicación y la confianza política continúa vigente y en constante evolución, con miras a continuar aportando evidencias que vayan más allá de una dicotomía entre videomalestar o movilización, y se enriquezcan nuevas líneas investigación que consideren aspectos adecuados a la evolución tanto de las sociedades democráticas, como de los medios de comunicación que en ellas se desarrollan.

\section{Diseños metodológicos en el estudio de los medios de comuni- cación y la confianza política}

En la literatura revisada sólo se encontraron estudios de carácter cuantitativo, siendo las encuestas la herramienta más utilizada. La mayoría realiza análisis de series temporales basados en datos provenientes de encuestas cara a cara o por entrevista telefónica. También es usual la utilización de experimentos que miden la influencia de estímulos de los medios en el nivel individual de desafección (Luengo \& Maurer, 2009). En una buena cantidad de los casos revisados, se utilizan datos secundarios de encuestas nacionales, ya sea para analizar datos de un país (Aruguete \& Muñiz, 2012; Ávila, 2014; Díaz \& Muñiz, 2017; Yang, et al., 2014); de un Estado (Lee, 2005; McLeod \& McDonald, 1985; Rodríguez-Virgili, López-Escobar, \& Tolsá, 2011; Ulbig \& Dunaway, 2008) o bien de varios países, en un diseño comparado (Ceron \& Memoli, 2015; Luengo \& Maurer, 2009; Torcal \& Maldonado, 2014). Por otro lado, un número menor de estudios utiliza experimentos (Botan, et al., 2016; Valentino, Beckmann, \& Buhr, 2001). 
De igual modo, es menor la cantidad de estudios encontrados que realizan de manera complementaria un análisis de contenido sobre la cobertura mediática, particularmente en radio y televisión, relacionando esos resultados con los datos obtenidos a través de las encuestas (Aarts, et al., 2010; Berganza, 2008; Ceron \& Memoli, 2015; Kleinnijenhuis, et al., 2006).

Respecto a la operacionalización de las variables, el análisis de la literatura ubica como variable independiente la exposición a noticias. Para medirla, se pregunta a los encuestados cuántos días por semana vieron un programa de noticias o leyeron un periódico, por lo que la respuesta se mide en una escala del o al 7 (Shehata, 2014; Shen \& Guo, 2013). Strömbäck et al. (2016) sugieren preguntar además si el periódico se leyó impreso o en línea y la misma diferenciación puede aplicarse a los programas de noticias que, además de verse en televisión, también están disponibles en portales de Internet. De igual manera se pregunta específicamente el nombre del noticiario o periódico (Aarts, et al., 2010), con el fin de establecer relaciones con la información obtenida en el análisis de contenido, así como valorar las diferencias entre quienes consumen cierto tipo de prensa, televisión (Newton, 1999) o noticias vía web. En algunos casos se enlistan los programas de noticias y periódicos que fueron incluidos en el análisis de contenido de medios, para que los encuestados indiquen cuántos días por semana se expusieron a noticias en ellos (Schuck, Boomgaarden, \& de Vreese, 2013).

La confianza política es una variable dependiente en estos estudios y se mide a un nivel tanto global como pormenorizado. En el primer caso, se pide que los encuestados evalúen la confianza que tienen en organizaciones y actores políticos como el parlamento o congreso, el gobierno y los políticos, el presidente, la burocracia, los tribunales, la policía, las autoridades electorales, entre otros, en una escala que va desde nada hasta mucho (Aarts et al., 2010; Ariely, 2015; Díaz \& Muñiz, 2017; Newton, 1999; Shehata, 2014; Shen \& Guo, 2013; Strömbäck et al., 2016; Tsfati et al., 2009; Tworzecki \& Semetko, 2012)

Por su parte, las variables de control reportadas en los estudios consideran aspectos sociodemográficos como la edad, el género, la escolaridad, el nivel de ingresos y la posición política, aunque en otros casos se busca controlar también variables como la conversación política, es decir si se discute la campaña con familia y amigos (Díaz \& Muñiz, 2017; Jebril, Albæk, \& De Vreese, 2013; Saldierna \& Muñiz, 2015; Schuck et al., 2013). En una buena parte de los trabajos que reportan estas variables de control, los jóvenes, personas del sexo masculino y aquellos con mayor nivel de educación confían más en las instituciones, mientras a mayor identificación partidista y mayor edad se incrementa la desconfianza.

También se contempla el interés político, preguntando directamente a los encuestados qué tan interesados están en política, con alternativas de respuesta que varían de no interesado a muy interesado (Newton, 1999; Shehata, 2014; Strömbäck \& Shehata, 2010; Tworzecki \& Semetko, 2012), así como el interés en la campaña, con la misma escala. El conocimiento político, de acuerdo con otros estudios (Aarts et al., 2010; Díaz \& Muñiz, 2017; Moy, Torres, Tanaka, \& McCluskey, 2005; Newton, 1999) se mide con preguntas sobre la vida política nacional, que incluyen identificar líderes políticos, 
políticas partidarias, cargos por los que se competía en el periodo electoral estudiado y conocimiento sobre organizaciones políticas y sus funciones.

\section{Apuntes finales}

Aunque existe una rica tradición de estudios sobre la confianza política y ésta ha sido continuamente abordada desde diversas disciplinas, nuestro trabajo ha buscado brindar una revisión sobre el vínculo de este objeto con la comunicación política, en vista de que se trata de una relación pertinente para una exploración más extensa, especialmente en las democracias latinoamericanas, donde la investigación con mayor solidez empírica aún está en ciernes. Como prueba de ello, hasta 2017 la base de datos Scopus no registraba trabajos provenientes de Latinoamérica, lo que pone en evidencia tanto el parroquialismo de la academia occidental, que excluye regiones enteras en investigaciones que aspiran a ser generalizadoras, como la necesidad de robustecer el trabajo empírico que se hace en los países de América Latina, de modo que permita alcanzar el nivel necesario para ganar circulación internacional a través de las plataformas de indexación.

En términos generales, la literatura revisada asume, y por lo general demuestra, que el tratamiento que los medios de comunicación brindan a los temas políticos afecta positiva o negativamente la confianza de la ciudadanía, lo mismo hacia los actores que hacia las instituciones políticas. Con ello no se demerita la influencia del resto de las variables que también han sido importantes y ampliamente tratadas en la literatura, sino que se ha elegido un área de conocimiento con una tradición teórica y empírica que también ha demostrado incidencia sobre la variable de confianza política.

Con todo, existen determinadas lagunas de conocimiento o procedimiento metodológico que constituyen áreas de oportunidad o expansión para esta línea. La primera de ellas se relaciona con la ausencia de trabajos que utilicen técnicas cualitativas, como grupos focales o entrevistas. Con ello se pierde una valiosa perspectiva subjetiva respecto a una cualidad tan vivencial como la confianza, al tiempo que se restringe la posibilidad de captar inductivamente nuevos fenómenos o manifestaciones de la misma, y con ello, refinar o innovar teóricamente. Explorar la relación entre confianza política y medios de comunicación utilizando métodos y técnicas interpretativistas, podría ser una perspectiva a considerar en estudios posteriores.

Otra área de oportunidad consiste en realizar una categorización de los objetos de confianza para precisar su medición. Si bien se citan diversos actores e instituciones en los reactivos, distinguirlos a través de su agrupación en niveles de gobierno o tipos de instituciones podría brindar una forma de medición más específica de cómo cada nivel se ve afectado y una explicación más profunda de los aspectos que inciden en sus variaciones. Observamos también la necesidad de abundar en estudios que den cuenta de cómo la confianza política ha variado a lo largo del tiempo en esta región del mundo y hasta qué grado los medios de comunicación están relacionados con esos cambios, descartando de 
paso varios factores exógenos. Asimismo, una veta que se distingue para los estudios empíricos es la utilización de diseños metodológicos que vayan más allá del análisis de una encuesta, vinculando los datos de ésta con el análisis de contenido para brindar explicaciones más robustas.

Nuestro propósito es que el presente trabajo contribuya a recapitular el vínculo entre la confianza política y los medios de comunicación, enriquezca el debate sobre los horizontes teóricos y empíricos de esta relación y brinde referencias útiles para quienes se interesan en esta línea de investigación.

\section{Declaración de conflicto de intereses}

Los autores no informaron ningún posible conflicto de intereses.

\section{Fondos}

Este artículo se desprende del proyecto de investigación "Infoentretenimiento, encuadres periodísticos y desconfianza ciudadana" (Clave 256670), financiado por el Consejo Nacional de Ciencia y Tecnología (CONACYT) de México.

\section{Referencias}

Aarts, K., Fladmoe, A., \& Strömbäck, J. (2012). Media, political trust, and political knowledge: A comparative perspective. En T. Aalberg \& J. Curran (Eds.), How the media inform democracy: a comparative approach (pp. 98-118). Nueva York, EU: Routledge.

Aarts, K., \& Semetko, H. A. (2003). The divided electorate: Media use and political involvement. Journal of Politics, 65(3), 759-784. doi:10.1111/1468-2508.00211

Almond, G. A., \& Verba, S. (1963). The civic culture: Political attitudes and democracy in five nations. New Jersey, EU: Princeton University Press.

Ariely, G. (2015). Does commercialized political coverage undermine political trust?: Evidence across European countries. Journal of Broadcasting \& Electronic Media, 59(3), 438-455. doi:10.1080/08838151.2015.1055000

Aruguete, N., \& Muñiz, C. (2012). Hábitos comunicativos y política. Efectos en las actitudes políticas de la población mexicana. Anagramas-Rumbos y Sentidos de la Comunicación, 10(20), 129146. doi:10.22395/angr.v10n20a9

Avery, J. M. (2009). Videomalaise or virtuous circle? The influence of the news media on political trust. The International Journal of Press/Politics, 14(4), 410-433. doi:10.1177/1940161209336224

Ávila, C. (2014, octubre). ¿Videomalaise o círculo virtuoso? Los efectos del consumo de noticias en el interés y la confianza política en Chile. Ponencia presentada en el I Congreso Nacional de la Asociación Chilena de Investigadores en Comunicación Social, Pucón, Chile. 
Bauer, P. C., \& Fatke, M. (2014). Direct democracy and political trust: Enhancing trust, initiating distrust-or both? Swiss Political Science Review, 20(1), 49-69. doi:10.1111/spsr.12071

Berganza, M. R. (2008). Medios de comunicación,"espiral del cinismo” y desconfianza política. Estudio de caso de la cobertura mediática de los comicios electorales europeos. ZER: Revista de Estudios de Comunicación, (25), 121-139.

Blind, P. K. (2007, junio). Building trust in government in the twenty-first century: Review of literature and emerging issues. Ponencia presentada en el 7th Global Forum on Reinventing Government: "Building Trust in Government", Viena, Austria.

Boţan, M. (2013). Different types of conflict in the news and their impact on political trust: the cumulated effect of incivility and intrusiveness. Revista Română de Comunicare şi Relaţii Publice, 15(2), 49-57.

Botan, M., Corbu, N., \& Sandu, D. (2016). The Complicated Relation Between News Frames and Political Trust: A Case Study of Romania. Středoevropské politické studie, 18(2-3), 122-140. doi:10.5817/CEPSR.2016.23.122

Camaj, L. (2014). Media use and political trust in an emerging democracy: Setting the institutional trust agenda in Kosovo. International Journal of Communication, 8(1), 187-209.

Cappella, J. N., \& Jamieson, K. H. (1996). News frames, political cynicism, and media cynicism. The Annals of the American Academy of Political and Social Science, 546(1), 71-84. doi:10.1177/0002716296546001007

Catterberg, G., \& Moreno, A. (2006). The individual bases of political trust: Trends in new and established democracies. International Journal of Public Opinion Research, 18(1), 31-48. doi:10.1093/ijpor/edho81

Ceron, A., \& Memoli, V. (2015). Trust in Government and Media Slant A Cross-Sectional Analysis of Media Effects in Twenty-Seven European Countries. The International Journal of Press/Politics, 2O(3), 339-359. doi:10.1177/1940161215572634

Chen, X., \& Shi, T. (2001). Media effects on political confidence and trust in the People's Republic of China in the post-Tiananmen period. East Asia, 19(3), 84-118. doi:10.1007/s12140-0010011-3

Coleman, J. S. (1986). Social theory, social research, and a theory of action. American Journal of Sociology, 91(6), 1309-1335. doi:10.1086/228423

Corduneanu, I., \& Muñiz, C. (2011). ¿Autoritarismo superado? Medios y actitudes políticas en el contexto mexicano. En C. Muñiz (Coord.), Comunicación, Política y Ciudadanía. Aportaciones actuales al estudio de la comunicación política (pp. 283-307). Ciudad de México, México: Fontamara.

Cueto, F. (2007). Desconfianza política, instituciones y gobernabilidad democrática en la República Dominicana. Ciencia y Sociedad, 32(2), 249-280. doi:10.22206/cys.2007.v32i2.pp249-80

Dalton, R. J. (2017). Political trust in North America. En S. Zmerli \& T. W.G. van der Meer (Eds.), Handbook on Political Trust (pp. 375-394). Cheltenham, UK: Edward Elgar Publishing. doi:10.4337/9781782545118.00035

de Vreese, C. H., \& Elenbaas, M. (2008). Media in the game of politics: Effects of strategic metacoverage on political cynicism. The International Journal of Press/Politics, 13(3), 285309. doi:10.1177/1940161208319650 
Degirmenci, N. (2016). Politics is a dirty game!: A case study of political cynicism in Turkey. International Journal of Liberal Arts and Social Science, 4(9), 114-132.

del Tronco, J. (2012). Las causas de la desconfianza política en México. Perfiles Latinoamericanos, 2O(40), 227-251.

Díaz, O. F. (2017). Las campañas electorales, los medios y su impacto en el compromiso cívico de los mexicanos en la elección presidencial de 2012. Comunicación y Sociedad, (29), 139-164. doi:10.32870/cys.voi29.6302

Díaz, O. F., \& Muñiz, C. (2017). Los efectos de la comunicación política en el compromiso político de los jóvenes en la elección presidencial mexicana de 2012. Revista Mexicana de Ciencias Políticas y Sociales, 61(229), 181-221. doi:10.1016/So185-1918(17)30008-9

Dogan, M. (2005). Erosion of confidence in thirty European democracies. Comparative Sociology, 4(1-2), 10-53. doi:10.1163/1569133054621932

Floss, D. (2008). Mass media's impact on confidence in political institutions: The moderating role of political preferences. A preferences-perceptions model of media effects. National Centre of Competence in Research (NCCR) Challenges to Democracy in the 21st Century (Working Paper no. 26), Universidad de Zurich. doi:10.5167/uzh-94934

García, Ó., \& Ruiz, J. (2007, septiembre). Media and political disaffection in México and Spain. Ponencia presentada en el VIII Congreso Español de Ciencia Política y de la Administración Política, Valencia, España.

Gómez, L. L. H. (2011). Confianza en las instituciones electorales en méxico: el ife bajo la mirada ciudadana. Revista Debates, 5(1), 9-23. doi:10.22456/1982-5269.20374

González, S. (2006). Desconfianza política: el colapso del sistema de partidos en Venezuela. En R. Cornejo Bustamante \& A. Román Zavala (Coords.), En los intersticios de la democracia y el autoritarismo. Algunos casos de Asia, África y América Latina (pp. 172-215). Buenos Aires, Argentina: CLACSO.

Guerrero, M. A., Rodríguez-Oreggia, E., \& Machuca, C. (2014). Consumo informativo y culturas cívicas: el papel de internet y las audiencias proactivas. En G. Meixueiro \& A. Moreno (Coords), El comportamiento electoral mexicano en las elecciones de 2012 (pp. 229-265). Ciudad de México, México: ITAM y CESOP.

Guggenheim, L., Kwak, N., \& Campbell, S. W. (2011). Nontraditional news negativity: The relationship of entertaining political news use to political cynicism and mistrust. International Journal of Public Opinion Research, 23(3), 287-314. doi:10.1093/ijpor/edro15

Hakhverdian, A., \& Mayne, Q. (2012). Institutional trust, education, and corruption: A micro-macro interactive approach. The Journal of Politics, 74(3), 739-750. doi:10.1017/Soo22381612000412

Hallin, D. C., \& Mancini, P. (2004). Comparing media systems: Three models of media and politics. New York, EU: Cambridge University Press.

Herman, T. (2010). The emergence of antipolitical sentiment in Israel. Recuperado de http://www.idi.org.il/sites/english/ResearchAndPrograms/PoliticsandAntiPolitics/Pages/ TheEmergenceofAntipolitics.aspx

Hetherington, M. J. (1998). The political relevance of political trust. American Political Science Review, 92(4), 791-808. doi:10.2307/2586304 
Hetherington, M. J., \& Husser, J. A. (2012). How trust matters: The changing political relevance of political trust. American Journal of Political Science, 56(2), 312-325. doi:10.1111/j.15405907.2011.00548.x

Hetherington, M. J., \& Rudolph, T. J. (2008). Priming, performance, and the dynamics of political trust. The Journal of Politics, $70(2), 498-512$. doi:10.1017/s0022381608080468

Hibbing, J. R., \& Theiss-Morse, E. (1995). Congress as public enemy: Public attitudes toward American political institutions. New York, EU: Cambridge University Press.

Holtz-Bacha, C. (1990). Videomalaise revisited: Media exposure and political alienation in West Germany. European Journal of Communication, 5(1), 73-85. doi:10.1177/0267323190005001005

Iyengar, S., \& Kinder, D. R. (2010). News that matters: Television and American opinion. Chicago, EU: University of Chicago Press.

Jackman, R. W., \& Miller, R. A. (1996). A renaissance of political culture? American Journal of Political Science, 4O(3), 632-659. doi:10.2307/2111787

Jebril, N., Albæk, E., \& de Vreese, C. H. (2013). Infotainment, cynicism and democracy: The effects of privatization vs personalization in the news. European Journal of Communication, 28(2), 105-121. doi:10.1177/0267323112468683

Kanji, M. (2002). Political discontent, human capital, and representative governance in Canada. En N. Nevitte (Ed.), Value change and governance in Canada (pp. 71-106). Toronto, Canadá: University of Toronto Press. doi:10.3138/9781442683006-007

Kleinnijenhuis, J., Van Hoof, A. M., \& Oegema, D. (2006). Negative news and the sleeper effect of distrust. The International Journal of Press/Politics, 11(2), 86-104. doi:10.1177/1081180x06286417

Knorn, W. (2016, noviembre). Political trust as a variable or as a process? A methodological question. Ponencia presentada en la Conferencia regional de la World Association for Public Opinion Research (WAPOR), Barcelona, España.

Latinobarómetro. (2016). El declive de la democracia. Informe Latinobarómetro 2016. Recuperado de http://www.latinobarometro.org

Lee, T.-T. (2005). Media effects on political disengagement revisited: A multiple-media approach. Journalism \& Mass Communication Quarterly, 82(2), 416-433. doi:10.1177/107769900508200211

Levi, M., \& Stoker, L. (2000). Political trust and trustworthiness. Annual Review of Political Science, 3(1), 475-507. doi:10.1146/annurev.polisci.3.1.475

Loveless, M. (2010). Understanding media socialization in democratizing countries: Mobilization and malaise in Central and Eastern Europe. Comparative Politics, 42(4), 457-474. doi:10.5129/o01041510x12911363510114

Luengo, Ó., \& Coimbra-Mesquita, N. (2013). Medios y desafección política: Un estudio comparado de Brasil y España. Comunicación y Sociedad, 26(1), 115-128. doi:10.15581/o03.26.1.117-132

Luengo, Ó., \& Maurer, M. (2009). A virtuous circle for all? Media exposure and political trust in Europe. CONfines de Relaciones Internacionales y Ciencia Política, 5(9), 39-48. 
McLeod, J. M., \& McDonald, D. G. (1985). Beyond simple exposure media orientations and their impact on political processes. Communication Research, 12(1), 3-33. doi:10.1177/009365085012001001

Mishler, W., \& Rose, R. (2005). What are the political consequences of trust? A test of cultural and institutional theories in Russia. Comparative Political Studies, 38(9), 1050-1078. doi:10.1177/0010414005278419

Morales, M. (2008). Evaluando la confianza institucional en Chile: Una mirada desde los resultados LAPOP. Revista de Ciencia Política, 28(2), 161-186. doi:10.4067/s0718090x2008000200007

Moreno, A. (2008). La opinión pública mexicana en el contexto postelectoral de 2006. Perfiles latinoamericanos, 16(31), 39-63.

Moreno, A. (2010). La confianza en las instituciones: consideraciones teóricas y metodológicas. En A. Moreno (Coord), La confianza en las instituciones: México en perspectiva comparada (pp. 11-59). Ciudad de México, México: CESOP.

Moy, P., \& Pfau, M. (2000). With malice toward all?: The media and public confidence in democratic institutions. Westport, Connecticut, EU: Greenwood Publishing Group.

Moy, P., \& Scheufele, D. A. (2000). Media effects on political and social trust. Journalism \& Mass Communication Quarterly, 77(4), 744-759. doi:10.1177/107769900007700403

Moy, P., Torres, M., Tanaka, K., \& McCluskey, M. R. (2005). Knowledge or trust? Investigating linkages between media reliance and participation. Communication Research, 32(1), 59-86. doi:10.1177/0093650204271399

Mutz, D. C., \& Reeves, B. (2005). The new videomalaise: Effects of televised incivility on political trust. American Political Science Review, 99(1), 1-15. doi:10.1017/sooo3055405051452

Newton, K. (1999). Mass media effects: mobilization or media malaise? British Journal of Political Science, 29(4), 577-599. doi:10.1017/s0007123499000289

Newton, K. (2006). May the weak force be with you: The power of the mass media in modern politics. European Journal of Political Research, 45(2), 209-234. doi:10.1111/j.14756765.2006.00296.x

Newton, K., \& Norris, P. (2000). Confidence in public institutions: Faith, culture or performance? En S. Pharr \& R. Putnam (Eds.), Disaffected democracies: What's troubling the trilateral countries (pp. 52-73) New Jersey, EU: Princeton University Press. doi:10.1515/9780691186849-007

Norris, P. (2000). A virtuous circle: Political communications in postindustrial societies. New York, EU: Cambridge University Press. doi:10.1017/cbo9780511609343

Norris, P. (2011). Democratic deficit: Critical citizens revisited. Cambridge, RU: Cambridge University Press.

Patterson, T. E. (1993). Out of order: How the decline of the political parties and the growing power of the news media undermine the American way of electing presidents. Nueva York, EU: Alfred Knopf.

Pereira, M. (2000). La crisis de confianza en la política y sus instituciones: los medios y la legitimidad de la democracia en Brasil. América Latina Hoy, 1(25), 23-33. 
Perloff, R. M. (2013). The dynamics of political communication: Media and politics in a digital age. New York, EU: Routledge.

Putnam, R. D., Leonardi, R., \& Nanetti, R. Y. (1994). Making democracy work: Civic traditions in modern Italy. Princeton, EU: Princeton university press.

Robinson, M. J. (1976). Public affairs television and the growth of political malaise: The case of "The Selling of the Pentagon”. American Political Science Review, 70(2), 409-432. doi:10.2307/1959647

Rodríguez-Virgili, J., López-Escobar, E., \& Tolsá, A. (2011). La percepción pública de los políticos, los partidos y la política, y uso de medios de comunicación. Comunicación y Sociedad, 24(2), 740.

Rosanvallon, P. (2006). Democracia y desconfianza. Revista de Estudios Políticos, (134), 219-237.

Rosanvallon, P. (2007). La contrademocracia: la política en la era de la desconfianza. Buenos Aires, Argentina: Ediciones Manantial.

Saldierna, A. R., \& Muñiz, C. (2015). Influencia de los hábitos comunicativos en la generación de participación y cinismo político entre los preciudadanos. "Disertaciones" Anuario electrónico de estudios en Comunicación Social, 8(2), 37-59. doi:10.12804/disertaciones.02.2015.03

Santiago Juárez, R. (2010). Crisis de legitimidad de las instituciones democráticas. Revista de Derecho, (33), 222-246.

Schmitt-Beck, R. (2015). Struggling up the hill: Short-term effects of parties' contacting, political news and everyday talks on turnout. Party Politics, 22(2), 227-240. doi:10.1177/1354068815605675

Schuck, A. R., Boomgaarden, H. G., \& de Vreese, C. H. (2013). Cynics all around? The impact of election news on political cynicism in comparative perspective. Journal of Communication, 63(2), 287-311. doi:10.1111/jcom.12023

Seyd, B. (2016, marzo). How should we measure political trust? Ponencia presentada en la Political Studies Association 66th annual international conference: "Politics and the Good Life", Brighton, Inglaterra.

Shehata, A. (2014). Game frames, issue frames, and mobilization: Disentangling the effects of frame exposure and motivated news attention on political cynicism and engagement. International Journal of Public Opinion Research, 26(2), 157-177. doi:10.1093/ijpor/edto34

Shen, F., \& Guo, Z. S. (2013). The last refuge of media persuasion: News use, national pride and political trust in China. Asian Journal of Communication, 23(2), 135-151. doi:10.1080/01292986.2012.725173

Strömbäck, J., \& Shehata, A. (2010). Media malaise or a virtuous circle? Exploring the causal relationships between news media exposure, political news attention and political interest. European Journal of Political Research, 49(5), 575-597. doi:10.1111/j.14756765.2009.01913.x

Strömbäck, J., Djerf-Pierre, M., \& Shehata, A. (2016). A question of time? A longitudinal analysis of the relationship between news media consumption and political trust. The International Journal of Press/Politics, 21(1), 88-110. doi:10.1177/1940161215613059

Torcal, M., \& Maldonado, G. (2014). Revisiting the dark side of political deliberation the effects of media and political discussion on political interest. Public Opinion Quarterly, 78(3), 679706. doi:10.1093/poq/nfuo35 
Tsfati, Y., Tukachinsky, R., \& Peri, Y. (2009). Exposure to news, political comedy, and entertainment talk shows: Concern about security and political mistrust. International Journal of Public Opinion Research, 21(4), 399-423. doi:10.1093/ijpor/edpo15

Tworzecki, H., \& Semetko, H. A. (2012). Media use and political engagement in three new democracies: Malaise versus mobilization in the Czech Republic, Hungary, and Poland. The International Journal of Press/Politics, 17(4), 407-432. doi:10.1177/1940161212452450

Ulbig, S. G., \& Dunaway, J. (2008, abril). Good news and bad news: The differential effects of media consumption on national and state-level political trust. Ponencia presentada en Annual meeting of the MPSA Annual National Conference, Chicago, Illinois, Estados Unidos.

Valentino, N. A., Beckmann, M. N., \& Buhr, T. A. (2001). A spiral of cynicism for some: The contingent effects of campaign news frames on participation and confidence in government. Political Communication, 18(4), 347-367. doi:10.1080/10584600152647083

Valentino, N. A., Buhr, T. A., \& Beckmann, M. N. (2001). When the frame is the game: Revisiting the impact of "strategic" campaign coverage on citizens' information retention. Journalism \& Mass Communication Quarterly, 78(1), 93-112. doi:10.1177/107769900107800107

Warren, M. E. (1999a). Democracy and trust. Cambridge, RU: Cambridge University Press. doi:10.1017/CBO9780511659959

Warren, M. E. (1999b). Democratic theory and trust. En M. E. Warren (Ed.), Democracy and trust (pp. 310-345). Cambridge, RU: Cambridge University Press. doi:10.1017/CBO9780511659959.011

Wilkins, K. (2000). The role of media in public disengagement from political life. Journal of Broadcasting \& Electronic Media, 44(4), 569-580. doi:10.1207/s15506878jobem4404_3

Yang, Y., Tang, M., Zhou, W., \& Huhe, N. (2014). the effect of media use on institutional trust in China. Problems of Post-Communism, 61(3), 45-56. doi:10.2753/PPC1075-8216610304

Zmerli, S., \& van der Meer, T. W. (2017). The deeply rooted concern with political trust. En S. Zmerli \& T. W. van der Meer (Eds.), Handbook on political trust (pp. 1-16). Northampton, Massachusetts, EU: Edward Elgar Publishing. doi:10.4337/9781782545118.00010 